\title{
Rate-dependent adhesion of viscoelastic contacts, Part I: Contact area and contact line velocity within model randomly rough surfaces
}

\author{
G. Violano ${ }^{\text {a,* }}$, A. Chateauminois ${ }^{\mathrm{b}}$, L. Afferrante ${ }^{\mathrm{a}}$ \\ ${ }^{a}$ Department of Mechanics, Mathematics and Management, Polytechnic University of Bari, Via E. Orabona, 4, 70125, Bari, Italy \\ ${ }^{\mathrm{b}}$ Soft Matter Science and Engineering Laboratory (SIMM), PSL Research University, UPMC Univ Paris 06, Sorbonne Universités, ESPCI Paris, CNRS, 10 rue \\ Vauquelin, 75231 Paris cedex 05, France
}

\section{A R T I C L E I N F O}

\section{Keywords:}

Viscoelasticity

Adhesion

Surface roughness

Energy release rate

\begin{abstract}
A B S T R A C T
In this work, we investigate dissipative effects involved during the detachment of a smooth spherical glass probe from a viscoelastic silicone substrate patterned with micro-asperities. As a baseline, the pull-off of a single asperity, millimeter-sized contact between a glass lens and a smooth poly(dimethylsiloxane) (PDMS) rubber is first investigated as a function of the imposed detachment velocity. From a measurement of the contact radius $a(t)$ and normal load during unloading phase, the dependence of the strain energy release rate $G$ on the velocity of the contact line $v_{c}=d a / d t$ is determined under the assumption that viscoelastic dissipation is localized at the edge of the contact. These data are incorporated into Muller's model (Muller, 1999) in order to predict the time-dependence of the contact size. Similar pull-off experiments are carried out with the same PDMS substrate patterned with spherical micro-asperities with a prescribed height distribution. From in situ optical measurements of the micro-contacts, scaling laws are identified for the contact radius $a$ and the contact line velocity $v_{c}$. On the basis of the observed similarity between macro and microscale contacts, a numerical solution is developed to predict the reduction of the contact radius during unloading.
\end{abstract}

\section{Introduction}

Adhesion is of paramount importance in the contact mechanics of micro and nano systems as, at the molecular scale, adhesive interactions between atoms are 'strong' compared to the usual forces acting between bodies (Kendall, 2007). However, adhesion is seldom observed at the macroscopic scale due to surface roughness, which reduces the area of actual contact. Nevertheless, when dealing with very soft matter (Young's modulus $E$ smaller than $0.5 \mathrm{MPa}$ ), strong adhesion may be still detected even in presence of surface roughness. Specifically, Tiwari et al. (2017) observed that for a PolyDiMethylSiloxane (PDMS) elastomer with Young's modulus $E \approx 0.02 \mathrm{MPa}$, the work of adhesion increases by nearly a factor of $\sim 2$ when pull-off experiments are performed on rough surfaces due to the complete contact that likely occurs at the interface. Complete contact that is instead prevented when PDMS samples with larger Young's modulus $(E \approx 2 \mathrm{MPa})$ are considered.

Soft matter adhesion finds applications in several fields, e.g., design of pressure-sensitive-adhesives (PSA) (Deplace et al., 2009), soft robots (Majidi, 2014), and new technologies inspired by biotribological systems (Gebeshuber, 2007).
In most of adhesion tests on soft compliant spheres (Lorenz et al., 2013; Tiwari et al., 2017), the measured detachment force is generally greatly in excess of that predicted by Johnson, Kendall \& Roberts (JKR) theory (Johnson et al., 1971) and the detachment process is observed to be dependent on the rate of separation (Greenwood and Johnson, 1981).

JKR theory applies for purely elastic spheres and under quasistatic conditions. In experimental investigations, the pull-off process unlikely obeys the quasi-static conditions because dynamic effects such as contact instabilities often occur. As a result, during pull-off, the effective work of adhesion $\Delta \gamma_{\text {eff }}$ is affected by the velocity $v_{\mathrm{c}}$ of the contact line. Namely, $\Delta \gamma_{\text {eff }}$ may be strongly increased with respect to the quasi-static value $\Delta \gamma_{0}$ due to the viscous dissipation, where $\Delta \gamma_{0}$ follows the well-known Dupré's equation $\Delta \gamma_{0}=\gamma_{1}+\gamma_{2}-\gamma_{12}$, being $\gamma_{1}, \gamma_{2}$ the adhesive energies of the two contacting surfaces and $\gamma_{12}$ the interaction term.

Gent and Schultz (GS) 1972 observed that viscous effects are exclusively located close to the crack tip. Maugis and Barquins (MB) 1978 proposed a generalization of JKR theory, showing that the dependence of $\Delta \gamma_{\text {eff }}$ on $v_{\mathrm{c}}$ can be expressed in terms of a dissipation function $f\left(v_{\mathrm{c}}, T\right)$ 
related to the viscoelastic properties of the material and depending on the contact line velocity $v_{\mathrm{c}}$ and the temperature $T$. In particular, MB showed that, for a given elastomer, the effective work of adhesion $\Delta \gamma_{\text {eff }}$ is a universal function of $v_{\mathrm{c}}$. Moreover, performing experimental tests on three different geometries (spheres, punches and tapes (peeling)), MB found that the dependence of $\Delta \gamma_{\text {eff }}$ on $v_{\mathrm{c}}$ is not affected by the geometry and loading system. In MB's solution, viscous effects are assumed not involving bulk deformations as "gross displacements must be elastic for $G$ to be valid in kinetic phenomena", being $G$ the energy release rate, i.e., the amount of energy required to advance a fracture plane by a unit area. Robbe-Valloire and Barquins (1998) extended MB studies performing adherence experiments between a rigid cylinder and an elastomeric solid. They confirmed the existence of a master curve for $f\left(v_{\mathrm{c}}, T\right)$. Specifically, their results "prove once again that the master curve drawn and its variation... is a characteristic of the propagation in mode I at the interface of our rubber-like material, when viscoelastic losses are closely limited to the crack tip, so that $G$ can be calculated from the theory of linear elasticity."

More recently, Muller (1999) showed that the process of detachment of viscoelastic spheres can be described by a first-order differential equation, whose solution is based on the assumption originally proposed in Gent and Schultz (1972). Alternative approaches taking into account bulk deformations were proposed by the group of Barthel (Barthel and Haiat, 2002; Haiat et al., 2003; Barthel and Frétigny, 2009).

In this work, we present an experimental investigation of dissipative effects involved in the adhesion between a smooth spherical glass probe and a viscoelastic silicone substrate patterned with a prescribed height distribution of micrometer sized spherical asperities. Taking advantage from the fact that the size of these micro-asperities (radius of $100 \mu \mathrm{m}$ ) allows for an optical measurement of the space distribution of microcontact areas, such patterned surfaces obtained from micro-milling techniques were successfully used to probe the elastic interactions between micro-asperity contacts (Yashima et al., 2015) or to investigate adhesive equilibrium of rough contact interfaces (Acito et al., 2019). Here, we focus on the effects of viscoelastic dissipation on the ratedependence of micro-contact sizes during unloading at an imposed velocity using JKR-type experiments. The investigation of adhesive behavior at the level of micro-contact spots is inspired by the Roberts' statement (Roberts, 1979): "The contact of a smooth centimeter-sized rubber sphere may be regarded as that of a giant single asperity... The ability to predict the adhesion forces on a large asperity is a step towards building up a model of a real surface of micron-sized asperities, which approximate to an array of minute hemispheres of different heights and radii".

Accordingly, pull-off experiments have first been conducted on smooth PDMS surfaces to investigate the adhesion behavior at the macroscopic scale. Specifically, under the assumption of viscous effects located only near the detachment front, we propose a very simple methodology to calculate the time-dependent radius of the contacts by exploiting the Muller's approach (Muller, 1999) which was also used to calculate the adhesion hysteresis occurring in loading-unloading tests performed on smooth viscoelastic spheres (Violano and Afferrante, 2019a). Then, this approach is successfully extended at the micro-scale with no need to incorporate a size-dependence in the relationship ruling the dependence of the strain energy release rate on the velocity of the contact line.

\section{Detachment of viscoelastic spheres}

In order to detach a soft body from a rigid substrate, the energy release rate $G$ must be larger than the effective work of adhesion. In viscoelastic contacts, Gent and Schultz (1972) found

$G-\Delta \gamma_{0}=\Delta \gamma_{0} f\left(v_{\mathrm{c}}, T\right)$

where $\Delta \gamma_{0}$ is the adiabatic work of adhesion and $\Delta \gamma_{0} f\left(v_{\mathrm{c}}, T\right)$ is the drag due to viscoelastic losses at the crack tip, being $v_{\mathrm{c}}=-d a / d t$ the velocity of the contact line. The above relation usually works for $v_{\mathrm{c}}$ ranging from $10^{-5} \mathrm{~cm} / \mathrm{s}$ to $1 \mathrm{~cm} / \mathrm{s}$ (Andrews and Kinloch, 1973; Gent and Petrich, 1969; Kendall, 1973; Roberts, 1979) and allows to predict the kinetics of detachment (see Maugis and Barquins, 1978).

The function $f\left(v_{\mathrm{c}}, T\right)$, which is found to be independent of the geometry and loading system, can be described by the phenomenological equation

$f\left(v_{\mathrm{c}}, T\right)=k\left(a_{\mathrm{T}} v_{\mathrm{c}}\right)^{n}$,

where $k$ and $n$ are characteristic constants of the material and $a_{\mathrm{T}}$ is the William-Landel-Ferry (WLF) factor (Williams et al., 1955) accounting for the dependence of $f\left(v_{\mathrm{c}}, T\right)$ on the temperature $T$. Notice $k$ has dimensions of $(\mathrm{m} / \mathrm{s})^{-n}$, while $n$ and $a_{\mathrm{T}}$ are dimensionless factors.

Eq. (2) also accounts for the dependence of $G$ on the relaxed elastic modulus $E$ (Ramond et al., 1985), whose frequency dependence appears only at the crack tip (Charmet et al., 1998).

Introducing Eq. (2) in (1), we obtain

$G=\Delta \gamma_{0}\left[1+c v_{\mathrm{c}}{ }^{n}\right]$,

with $c=k a_{\mathrm{T}}{ }^{n}$.

For a given elastomer, the values of $c$ and $n$ can be obtained by fitting the experimental data relating $G$ and $v_{\mathrm{c}}$. As observed in Lorenz et al. (2013), the exponent $n$ "is not a universal number, but takes different values depending on viscoelastic modulus".

\subsection{Muller's model}

Muller (1999) proposed a two-parameter differential equation to describe the detachment of a viscoelastic sphere of radius $R$ and reduced Young's modulus $E^{*}$ from a rigid substrate

$\frac{d \bar{a}}{d \bar{\delta}}=\left[\frac{\Delta \gamma_{0}}{R E^{*}}\right]^{1 / 3} \frac{1}{\beta}\left[\bar{a}^{3}\left(1-\frac{\bar{\delta}}{3 \bar{a}^{2}}\right)^{2}-\frac{4}{9}\right]^{1 / n}$,

where $\bar{a}=a /\left[3 R\left(\pi \Delta \gamma_{0} /\left(6 E^{*} R\right)\right)^{1 / 3}\right]$ and $\bar{\delta}=\delta /\left[3 R\left(\pi \Delta \gamma_{0} /\left(6 E^{*} R\right)\right)^{2 / 3}\right]$ are the dimensionless contact radius and penetration, respectively, and the parameter $\beta$ is proportional to the driving velocity $V$

$\beta=\left(\frac{6}{\pi}\right)^{1 / 3}\left(\frac{4}{9} c\right)^{1 / n} V$.

Muller's model is based on two assumptions: (i) viscous effects are located exclusively near the crack tip; (ii) detachment occurs under constant $V$. The energy release rate $G$, which represents the effective work of adhesion $\Delta \gamma_{\text {eff }}$ required to break the contact, can be evaluated as

$G=\frac{\left(F_{\mathrm{H}}-F\right)^{2}}{6 \pi R F_{\mathrm{H}}}$

where $F_{\mathrm{H}}=4 E^{*} a^{3} /(3 R)$ is the Hertzian load and $F$ is the applied load. Eq. (6) is only valid under the assumption of viscous effects concentrated at the crack tip (see, for example, Baek et al., 2017).

\section{Experimental set-up}

JKR-like tests were carried out between an optical spherical glass lens and rubber substrates. The glass indenter, which is assumed to be smooth, has a radius of curvature of $103.7 \mathrm{~mm}$. Rubber substrates are made of commercially available PDMS silicones. Samples were manufactured by cross-linking at $70{ }^{\circ} \mathrm{C}$ for 48 hours a mixture of Sylgard 184 and Sylgard 527 silicones (Dow Chemicals), with a 0.35:0.65 weight ratio. As detailed by Palchesko et al. (2012), mixing these two silicone elastomers in different ratios allows to tune the elastic modulus in between a few $\mathrm{kPa}$ and $3 \mathrm{MPa}$. As compared to raw Sylgard 184, the Young's modulus of the selected Sylgard 184:Sylgard 527 mixture ( $E=0.83 \mathrm{MPa}$, see below) was decreased by a factor of about 3.6, with the aim of enhancing the adhesion properties. In addition, crosslinking 


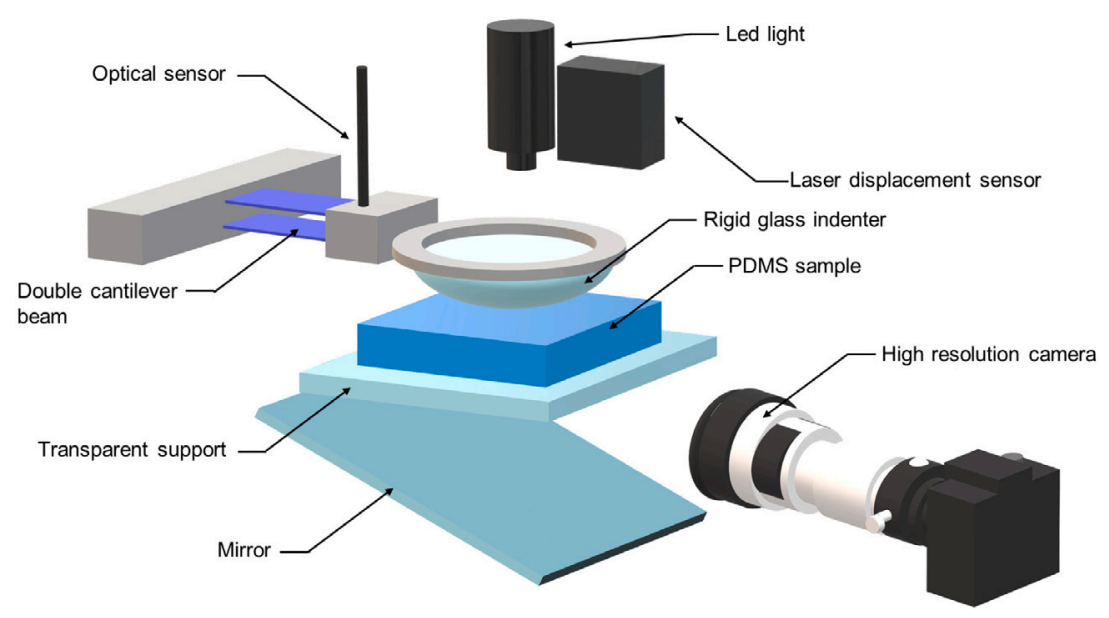

Fig. 1. Experimental setup of JKR adhesion tests.

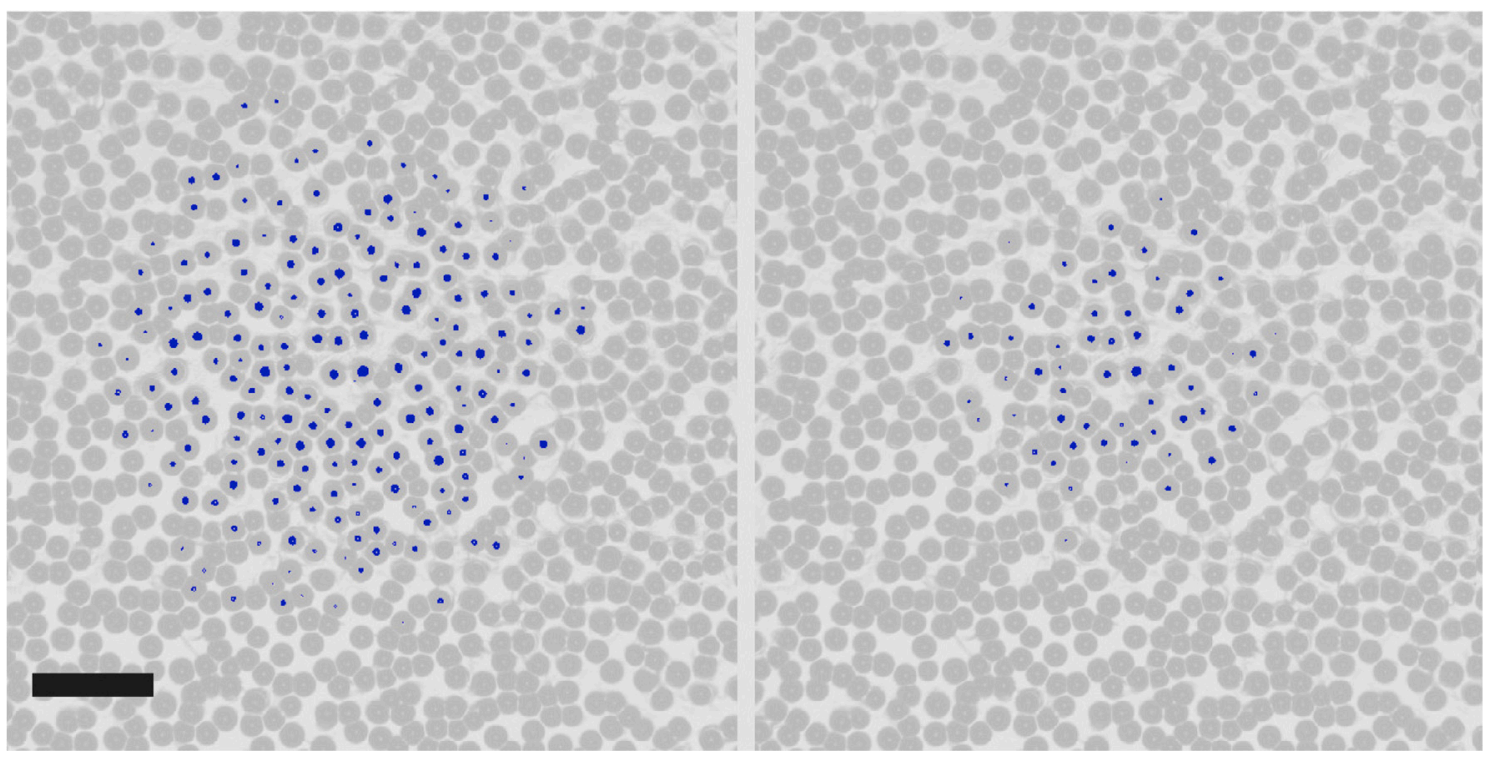

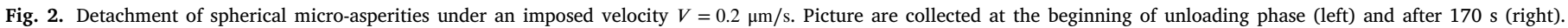
The contact spots (blue disks) are detected after post-processing of the contact pictures. The length of the black rectangle is $1 \mathrm{~mm}$.

simultaneously these two different silicone products was expected to result in an increased concentration of network defects such as dangling chains. As detailed in Palchesko et al. (2012), such imperfections are known to enhance the viscoelastic dissipation of silicone networks.

Fig. 1 shows a sketch of the experimental set-up. The spherical indenter is fixed to a motorized vertical translation stage by means of a double cantilever beam of known stiffness $\left(290 \mathrm{~N} \mathrm{~m}^{-1}\right)$. The value of the applied load is obtained from the deflection of the cantilever as it is measured using a high resolution optical sensor (Philtec D64-L). Due to the compliance of the double cantilever beam, the actual velocity of the lens can slightly differ from the prescribed velocity. In order to take account of this effect, a laser displacement sensor (Keyence LKH057) is used to monitor the actual position of the lens. The difference between the prescribed and actual velocities of the indenter was found to be significant only for the macroscopic single asperity contact close to pull-off, when the greatest tensile normal forces are achieved.

The PDMS sample is fixed to two crossed motorized linear translation stages, which allow to change the relative position of the rubber sample with respect to the indenter. A LED light spot is installed to illuminate in transmission the contact area. Once illuminated, contact pictures are recorded through the transparent PDMS using a zoom objective (Leica APO Z16) and a high resolution CMOS camera (SVS Vistek Exo, $2048 \times 2048$ pixels $^{2}$, 8 bits).

\subsubsection{Experiments on smooth samples}

The contact radius vs. load data obtained by indentation experiments on smooth PDMS samples were fitted according to JKR theory (Johnson et al., 1971) in order to evaluate the reduced elastic modulus $\left(E^{*}=0.83 \mathrm{MPa}\right)$ and the adhesion energy $\left(\Delta \gamma_{0}=0.037 \mathrm{~J} / \mathrm{m}^{2}\right)$. During the loading process, contact tests have been performed under controlled load conditions. Specifically, the applied load is increased step by step and, once each load step is reached, contact is maintained for a long time $(800 \mathrm{~s})$ to ensure that adhesive equilibrium is reached and viscoelastic effects are totally relaxed (Acito et al., 2019).

Unloading tests are performed at imposed driving velocity of the vertical stage, while continuously monitoring the lens position, the applied force, and the contact radius. Experiments are performed at three different values of the driving velocity $V=0.2,2,20 \mu \mathrm{m} / \mathrm{s}$. Three contact realizations have been carried out for each velocity.

\subsubsection{Experiments on rough samples}

PDMS samples were textured with a statistical distribution of spherical micro-asperities with the same radius of curvature. The patterned 


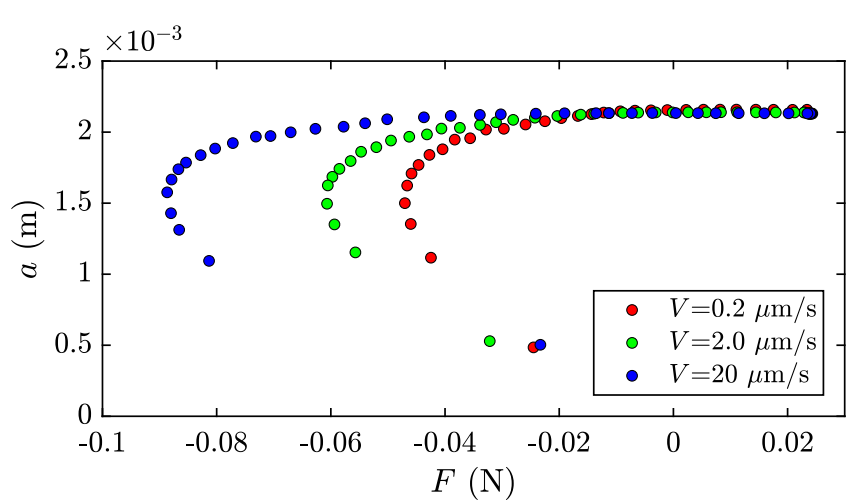

Fig. 3. Contact radius $a$ as a function of the applied load $F$. Results are shown for different unloading velocities of the indenter $V=0.2,2,20 \mu \mathrm{m} / \mathrm{s}$. For each velocity, three tests have been performed and the average values are reported in the plot.

surface was obtained by molding PDMS in PolyMethylMethAcrylate (PMMA) forms milled using ball-end mills with a radius of $100 \mu \mathrm{m}$. In order to enhance adhesive effects, a smoothening of the spherical cavities of the PMMA molds has been achieved by exposing them to a saturated $\mathrm{CHCl}_{3}$ vapor for 30 minutes. As detailed in Acito et al. (2019), such treatment leads to an increase in the radius of the spherical bumps up to a $10 \%$.

The patterned surface has been generated with a squared nominal area of $10 \mathrm{~mm}^{2}$, where asperities are randomly distributed with a density of $2 \times 10^{7} \mathrm{~m}^{-2}$. The spherical caps present heights distributed according to a Gaussian law with standard deviations $\sigma=5 \mu \mathrm{m}$.

Asperities are collocated with a non-overlapping constraint. For the considered surface density, each contacting asperity behaves as an isolated spherical punch and lateral interactions can be neglected (Yashima et al., 2015; Acito et al., 2019). We stress that such assumption is no longer valid when roughness on several length scales is considered like in the case of self-affine fractal geometries (Afferrante et al., 2018; Violano and Afferrante, 2019b,c).

Fig. 2 shows images of contact micro-spots (blue disks) captured during the unloading phase. Pulling tests have been performed at the same values of driving velocity $V$ used in the tests conducted on smooth samples $(V=0.2,2,20 \mu \mathrm{m} / \mathrm{s})$. Accurate measurements of the contact spots radii are achieved by standard thresholding techniques applied to the contact images after background removal. Namely, the measured radius $a_{i}$ of each micro-asperity contact is calculated as the square root of the $A_{i} / \pi$ ratio, where $A_{i}$ is the measured micro-contact area. It was verified that the value of the threshold does not significantly affect the measured values of $a_{i}$.

\section{Results}

\subsection{Smooth contact: macroscopic scale}

Fig. 3 shows the variation of the contact radius $a$ with the applied load $F$ occurring during pulling tests. Results are obtained for different values of the driving velocity $V$. Three contact tests were performed for each $V$ and the average values are reported in the plot.

The detachment process is clearly rate-dependent as pull-off force enhancement is observed by increasing $V$.

Fig. 4A shows, in a semilogarithmic representation, the reduction of the contact radius $a$ with the time $t$. Experimental data are fitted according to the following relation

$a(t)=p_{1} \sqrt{1-\left(\frac{t}{t_{\mathrm{po}}+1}\right)^{p_{2}}}$

where $t_{\mathrm{po}}$ is the instant at which detachment occurs and $p_{1}, p_{2}$ are fitting parameters.
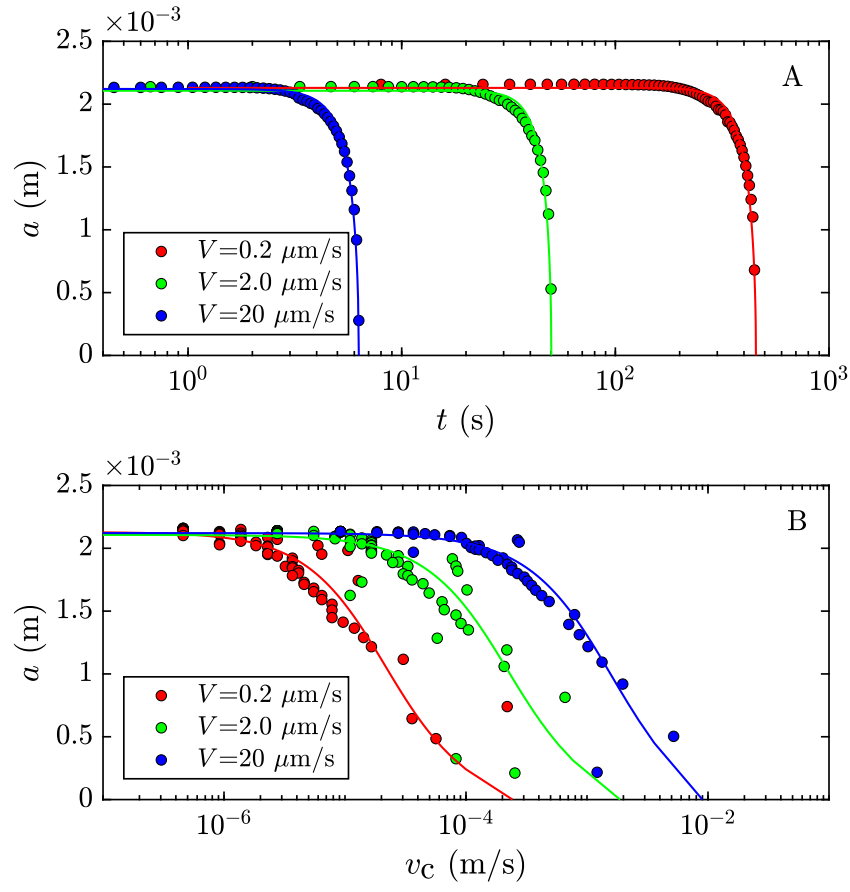

Fig. 4. (A) Contact radius $a$ as a function of the time $t$. Results show the average of three contact tests. Blue, green and red markers are referred to the unloading velocities of the indenter $V=0.2,2,20 \mu \mathrm{m} / \mathrm{s}$, respectively. Solid lines are the fit of experimental data made with Eq. (7) $\left(p_{1}=0.0021\right.$ and $p_{2}=5.93,7.15,7.89$ for $V=0.2,2,20 \mu \mathrm{m} / \mathrm{s}$, respectively). (B) Contact radius $a$ as a function of the crack tip velocity $v_{\mathrm{c}}$. Solid lines are obtained by taking derivation of fitted equations in Fig. A. (For interpretation of the references to color in this figure legend, the reader is referred to the web version of this article.)

During detachment, the contact line velocity can be easily obtained as $v_{\mathrm{c}}=-d a / d t$. Fig. $4 \mathrm{~B}$ shows the curves $a$ vs. $v_{\mathrm{c}}$ obtained in the experiments at different velocities. The maximum value of $v_{\mathrm{c}}$ is reached when abrupt pull-off occurs. Notice that when increasing $V$ of one order of magnitude the same enhancement in $v_{\mathrm{c}}$ is also observed.

The effective work of adhesion $G$ is calculated as a function of the measured contact line velocity by Eq. (6) using the experimental values of the normal load $F$. Deng and Kesari (2019) showed that the machine compliance can influence the adhesive behavior and the contact instability at pull-off. However, in a previous work, Barquins (1983) showed that Eq. (1) applies whatever the compliance of the testing machine and the instantaneous deformation imposed on the system. As a result, the $G\left(v_{\mathrm{c}}\right)$ relationship, which is the main input for the description of contact, is unaffected by the machine stiffness. Moreover, we have taken into account the actual stiffness of the system because the load $F$ is read from the deflection of the double cantilever beam. The same procedure has been recently used in Baek et al. (2017) in experiments of detachment of glass spherical lens from PDMS blocks.

Fig. 5 shows the quantity $\left(G-\Delta \gamma_{0}\right) / \Delta \gamma_{0}$ as a function of $v_{\mathrm{c}}$ in a double logarithmic chart. Markers denote experimental data, while the dotted black line is the fit obtained with Eq. (3) using $n=0.25$ and $c=31 \mathrm{~m} / \mathrm{s}^{-0.25}$. Maugis and Barquins (1978) found $n=0.6$ for a viscoelastic polyurethane rubber. More recently, Lorenz et al. (2013) found $n=0.19$ for polyurethane and $n=0.12$ for Sylgard 184 PDMS rubber. As discussed by Barthel and Frétigny (2009), the dependence of $G$ on the contact line velocity can be related to the viscoelastic creep function of the solids. Accordingly, the fact that we found for the used Sylgard 184:Sylgard 527 mixture an exponent $n$ greater than for raw Sylgard 184 probably reflects the enhanced viscoelastic dissipation of the PDMS mixture.

The values of $c$ and $n$ can be used in Eq. (5) to calculate the values of $\beta$ and then Eq. (4) can be numerically integrated to obtain $a(\delta)$. 


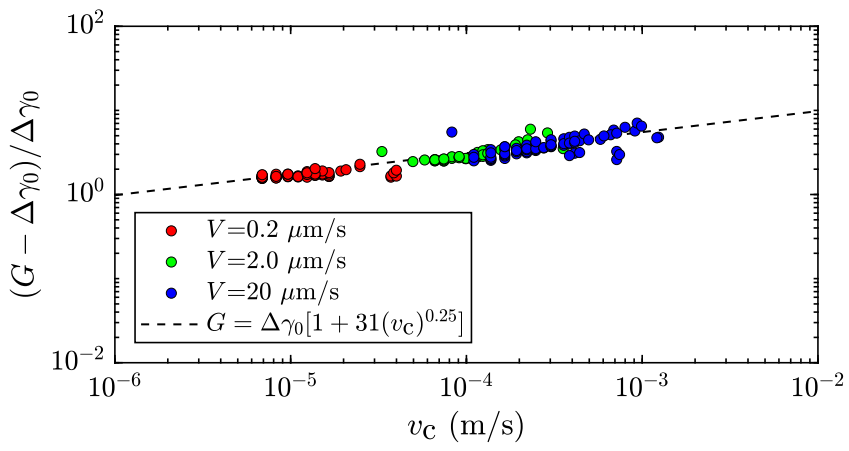

Fig. 5. Relative increase $\left(G-\Delta \gamma_{0}\right) / \Delta \gamma_{0}$ as a function of the crack tip velocity $v_{\mathrm{c}}$. Results are shown for three contact realizations. Blue, green and red markers are referred to the unloading velocities of the indenter $V=0.2,2,20 \mu \mathrm{m} / \mathrm{s}$. The dotted line is the fit obtained with Eq. (3). (For interpretation of the references to color in this figure legend, the reader is referred to the web version of this article.)

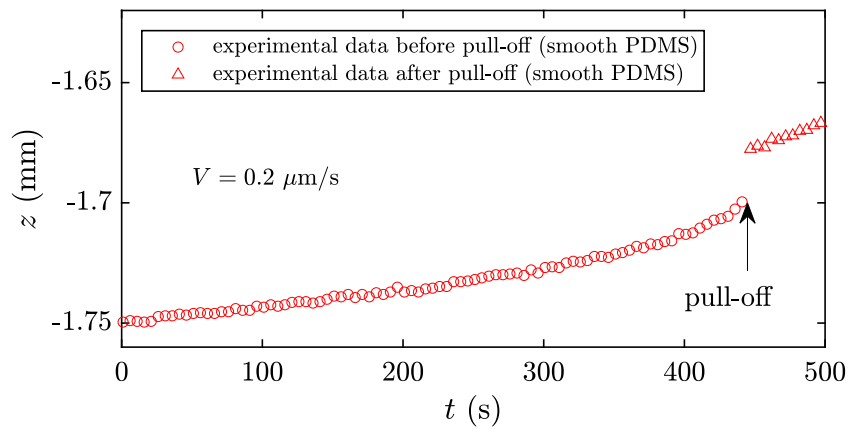

Fig. 6. Variation with time of the vertical position $z$ of the glass lens during unloading. Results are given for smooth case and imposed driving velocity $V=0.2 \mu \mathrm{m} / \mathrm{s}$.

Assuming that $V$ is constant during the unloading phase, the instant $t_{\mathrm{po}}$ at which pull-off occurs can be estimated by $t_{\mathrm{po}}=\left(\delta_{0}-\delta_{\mathrm{po}}\right) / V$, where $\delta_{0}$ is the initial penetration and $\delta_{\text {po }}$ the jump-off distance. However, in our experiments we found that the actual unloading velocity $V_{\text {act }}(t)$ is not constant as the spherical indenter is held to the stage using a compliant double cantilever beam. Due to the deflection of the beam, the velocity $V_{\text {act }}$ of the lens can be different from the imposed velocity $V$. This is especially true for the experiments on smooth PDMS, where high forces can be achieved. However, this effect is quantified by the laser displacement sensor, which monitors the actual position $z(t)$ of the lens. In this regard, Fig. 6 shows the variation of the vertical position $z$ of the glass lens with the time during the unloading phase for imposed velocity $V=0.2 \mu \mathrm{m} / \mathrm{s}$. The actual velocity $V_{\text {act }}$ is given by the slope of the curve and can be derived as $V_{\text {act }}(t)=\Delta z / \Delta t$.

In the original Muller's model, the unloading velocity $V=-d \delta / d t$ is assumed to be constant. However, we can modify Eq. (5) by introducing the actual velocity $V_{\text {act }}(\delta)$ in the parameter $\beta$

$\beta=\left(\frac{6}{\pi}\right)^{1 / 3}\left(\frac{4}{9} c\right)^{1 / n} V_{\text {act }}(\bar{\delta})$.

where $V_{\text {act }}(\delta)$ is obtained by interpolating experimental data.

The time required to move from $\delta_{0}$ to a generic $\delta$ is then calculated as

$t=\int_{\delta_{0}}^{\delta} \frac{d \delta^{\prime}}{V_{\text {act }}\left(\delta^{\prime}\right)}$

Results are shown in Fig. 7A-C, where the contact radius $a(t)$, normalized with respect to its initial value, is plotted against the time normalized with respect to the period required for pull-off to occur. Results are given for different unloading velocities and show a good agreement between experimental data and numerical predictions.

\subsection{Rough contact: microscopic scale}

In the experiments performed on rough samples the number of micro-asperities detected in contact at the end of the loading phase is around 160. However, for the sake of clarity, Fig. 8A and B show the variation of the contact radius $a$ of 8 micro-asperities in terms of the time and the contact line velocity $v_{\mathrm{c}}$, respectively. Results are shown for $V=0.2 \mu \mathrm{m} / \mathrm{s}$. In general, asperities with a larger value of the initial contact radius $a$ require a longer time to complete their detachment process.

Results on smooth and rough samples suggest the existence of scale effects on both contact radius $a$ and detachment front velocity $v_{\mathrm{c}}$. For this reason, we rescale the above results introducing the factors $s_{\mathrm{a}}=$ $a_{0 \text { macro }} / a_{0 i}, s_{\mathrm{t}}=t_{\mathrm{po}-\text { macro }} / t_{\mathrm{po}-i}$ and $s_{\mathrm{v}}=s_{\mathrm{a}} / s_{\mathrm{t}}$. The quantity $a_{0 \text { macro }}$ is the initial value of the contact radius measured at the macroscale on smooth PDMS samples at the end of the loading phase (when unloading starts); $a_{0 i}$ is instead the initial value of the contact radius detected for the $i$ th micro-asperity. Similarly, $t_{\text {po-macro }}$ is the time at which pull-off occurs at the macroscale (that is measured in the tests performed on smooth PDMS samples), while $t_{\mathrm{po}-i}$ is the time required (and measured in the tests on rough PDMS samples) to detach the $i$ th micro-asperity.

Therefore, contact radius $a$, contact line velocity $v_{\mathrm{c}}$ and time are rescaled with the above factors. The new curves are given in Fig. 9 for three different driving velocities $V$ and in a semi-log plot. Solid lines denote the curves obtained at the macroscopic scale in the experiments conducted on smooth substrate. Dashed lines identify the curves measured for each micro-asperity during the detachment tests performed on the rough PDMS samples. With the proposed scaling, the curves obtained on the contact microspots become comparable with the curves measured at the macroscale (smooth samples). Such result suggests that the distributions of the actual contact line velocities $v_{\mathrm{c}}$, which are achieved locally at micro-contact, scale during contact unloading. This, in turn, suggests that the parameters of Muller's model identified at the macroscale can be reasonably applied to the microcontacts.

Such an assumption finds also its motivation in recent results by Lorenz et al. (2013), who performed adhesion experiments on smooth spheres of different radii (ranging from $R \approx 3 \mathrm{~mm}$ to $R=46.5 \mathrm{~mm}$ ) and different materials. They deduced $\Delta \gamma_{\text {eff }}$ as a function of the contact line velocity $v_{\mathrm{c}}$ using JKR theory and observed that the experimental data exhibited the same velocity dependence as calculated by Eq. (1) for $v_{\mathrm{c}}<10^{-4} \mathrm{~m} / \mathrm{s}$ (which corresponds to the range of velocities measured in our experiments on micro-spots).

The same plots given in Fig. 7 are reported in Fig. 10 for each of the micro-contacts detected during the unloading phase. A satisfactory good agreement is found between experimental data and numerical predictions, which are obtained with the "macroscale" values of $c$ and $n$. Once again, we have introduced in the Muller's model the actual value of the unloading velocity, which is however constant and slightly lower than the imposed one $\left(V_{\text {act }}=0.8 \mathrm{~V}\right)$. In fact, as shown in Fig. 11, the measured values of the vertical position $z$ of the glass lens during the unloading phase are well fitted by a linear law whose slope gives $V_{\text {act }}$.

In the $a-t$ curves, we can identify an initial period where the contact line velocity is zero and the contact radius constant. Our data suggest this "stick time" $t_{\text {stick }}$ is slightly affected by the initial value $a_{0}$, as shown in Fig. 12, where $t_{\text {stick }}$ is plotted against $a_{0}$ in a semi-log chart. Notice we conventionally calculated the stick time at the point where the initial contact radius $a_{0}$ is reduced by $0.1 \%$. Although scatter in results does not allow to come up with a definitive conclusion on this point, we observe that our outcomes agree with results given in Baek et al. (2017), where the contact radius is found to be constant at the beginning of the unloading phase when increasing the contact penetration. Similar results are also found in Deruelle et al. (1998) from JKR experiments and in Haiat et al. (2003), where numerical calculations on the adhesive contact of linear viscoelastic spheres are performed. 

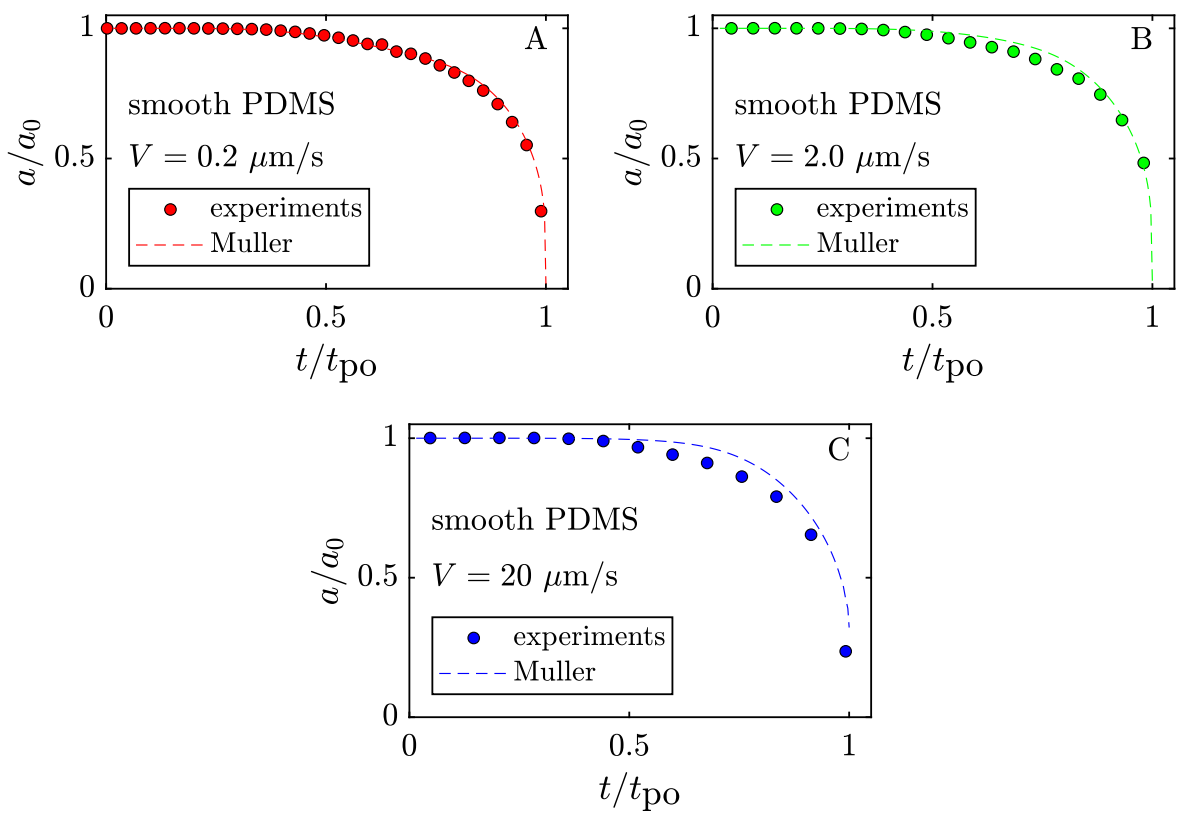

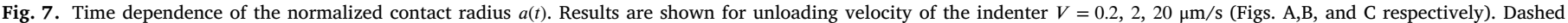
lines denote Muller's model predictions, while markers experimental data, which are averaged on three contact realizations.
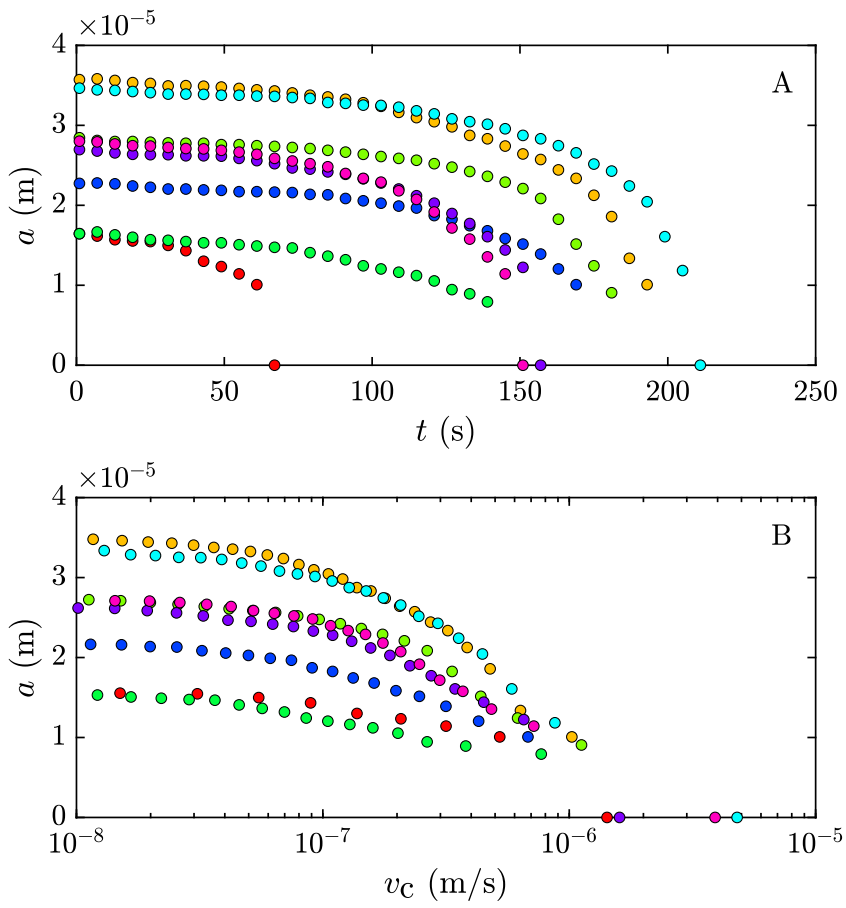

Fig. 8. (A) Contact radius $a$ of micro-asperities as a function of time $t$. Markers denote experimental data for a selection of eight different micro-contacts. Unloading tests are performed at $V=0.2 \mu \mathrm{m} / \mathrm{s}$. (B) Contact radius $a$ as a function of the crack tip velocity $v_{\mathrm{c}}$.

For the above reason, when increasing $a_{0}$, the $a-t$ curve does not scale homothetically and a master curve cannot be found. This explains the scatter in Fig. 10. However, an increasing trend of the pull-off time with $a_{0}$ can be observed in our experimental data, as shown in Fig. 13A$\mathrm{C}$, where results are presented for different unloading velocities. As the pull-off time is due to two contributions, the "stick time" and the "pulling-off time", the increasing trend of $t_{\mathrm{po}}$ with $a_{0}$ is basically due to the variation of $t_{\text {pulling-off }}$ as $t_{\text {stick }}$ is only a little affected by $a_{0}$. Anyway,
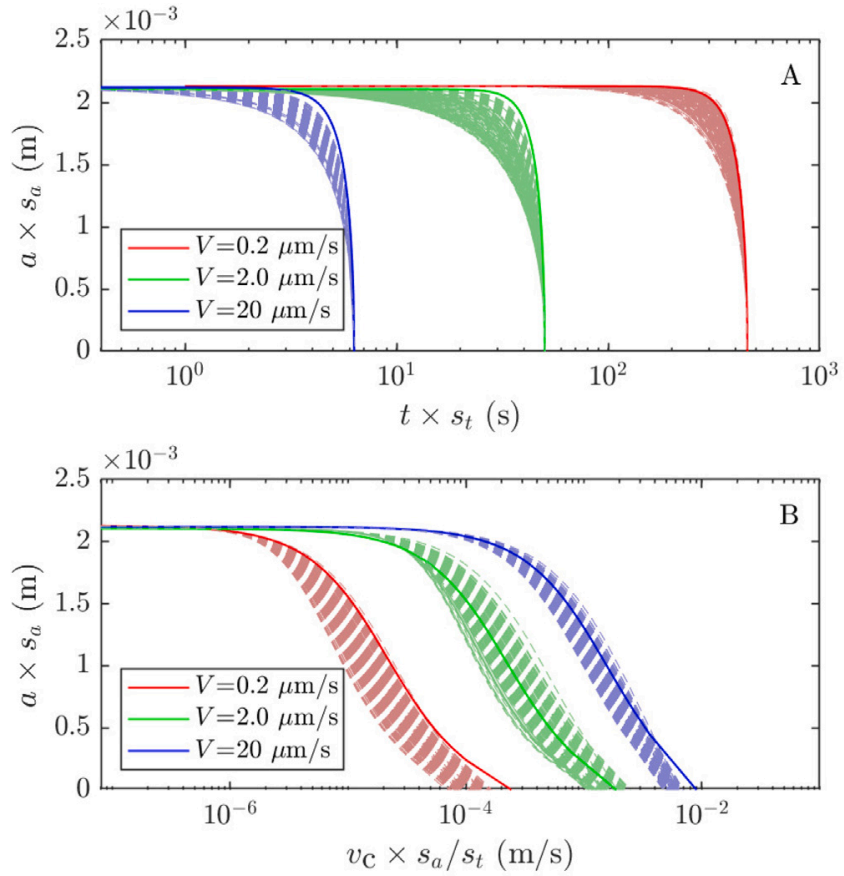

Fig. 9. (A) Contact radius $a \times s_{a}$ as a function of time $t \times s_{t}$ (semilog scale). Solid lines denote the smooth macro-spot detachment curve. Dashed lines denote the detachment curves of 160 micro-asperities. Red, green and blue curves refer to $V=0.2,2.0,20$, $\mu \mathrm{m} / \mathrm{s}$. (B) Contact radius $a \times s_{a}$ as a function of the contact line velocities $v_{\mathrm{c}} \times s_{a} / s_{t}$ (semilog scale). Legend symbols are the same of Fig. A. (For interpretation of the references to color in this figure legend, the reader is referred to the web version of this article.)

since data are strongly scattered because of the dynamic nature of the pull-off process, a clear trend cannot be easily identified.

\section{Conclusions}

In this paper, we have investigated the pull-off behavior of a rough contact interface between a smooth glass lens and a nominally flat 

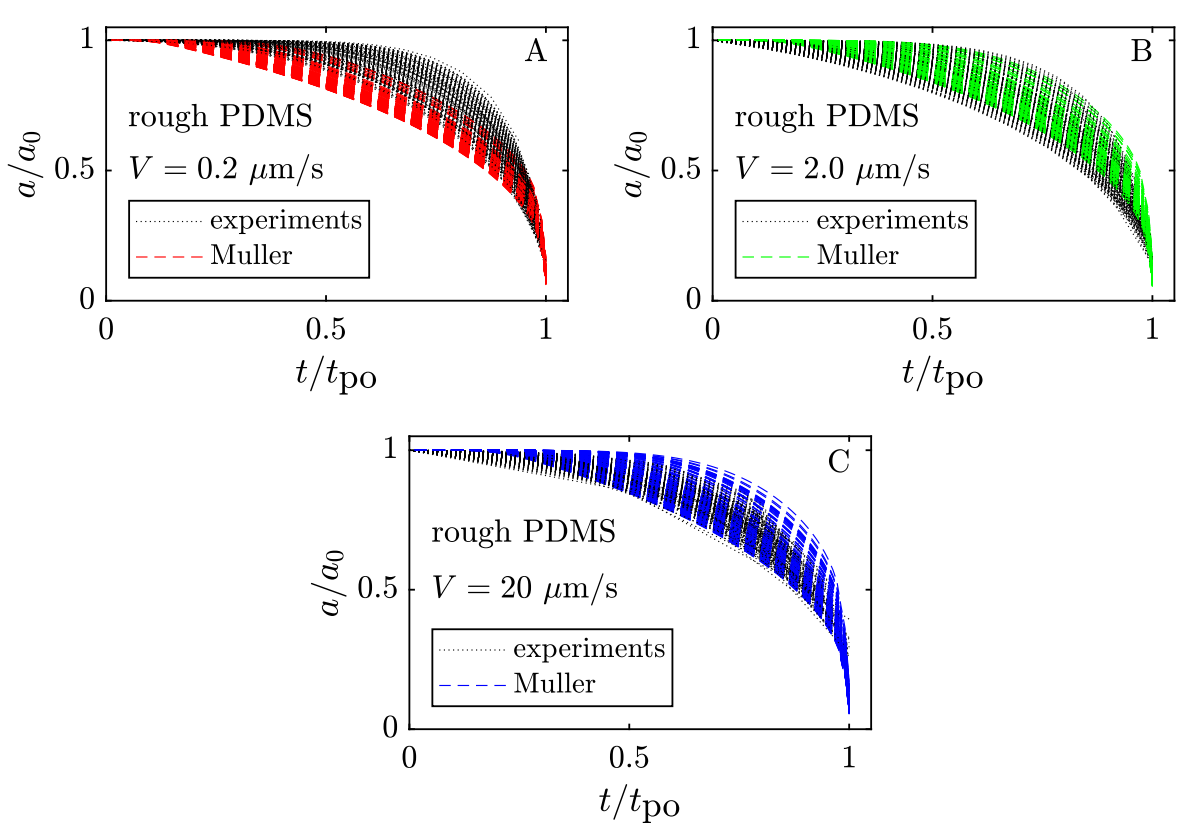

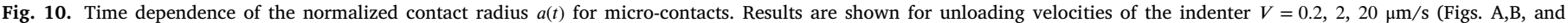
C respectively). Black dotted lines denote experimental data, while colored dashed lines, the Muller's model predictions.

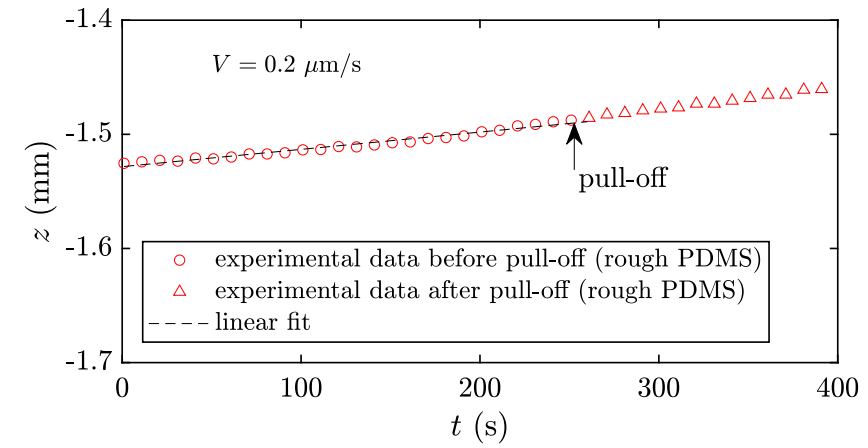

Fig. 11. Variation with time of the vertical position $z$ of the glass lens during unloading. Results are given for rough case and imposed driving velocity $V=0.2$ $\mu \mathrm{m} / \mathrm{s}$.

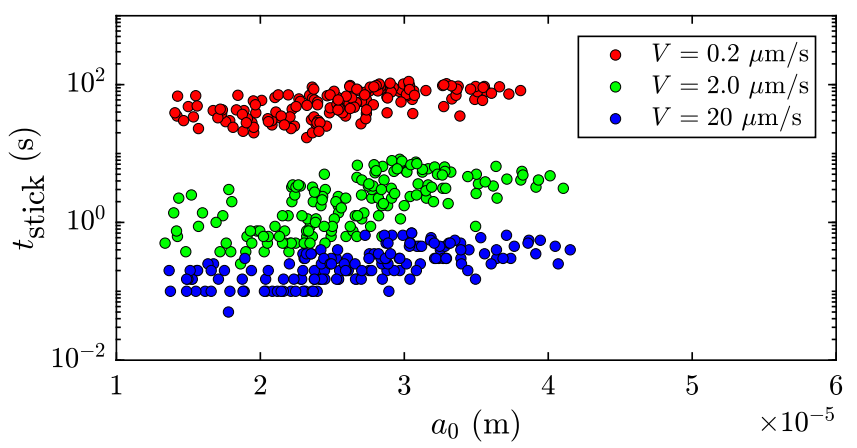

Fig. 12. Stick time $t_{\text {stick }}$ as a function of the initial contact radius $a_{0}$. Results are shown for rough PDMS and unloading velocities of the indenter $V=0.2,2,20 \mu \mathrm{m} / \mathrm{s}$.

viscoelastic substrate patterned with a height distribution of spherical micro-asperities. In the absence of any elastic coupling between microcontacts, this system allows to measure simultaneously the pull-off behavior of a collection of micro-asperities contacts differing in their initial (equilibrium) adhesive contact radius. From a comparison with macroscale pull-off experiments, it also offers the possibility to investigate the occurrence of scale effects in dissipative processes involved in adhesion.

Results show the contact radius almost scales according to the ratio $s_{\mathrm{a}}=a_{0 \text { macro }} / a_{0 \text { micro }}$, being $a_{0}$ the initial radius measured at the beginning of the unloading process. Similarly, the contact line velocity $v_{\mathrm{c}}$ is found scaling with a factor $s_{\mathrm{v}}$ depending on the ratio $s_{\mathrm{a}} / s_{\mathrm{t}}$, where $s_{\mathrm{t}}=t_{\mathrm{po}-\text { macro }} / t_{\mathrm{po}-\text { micro }}$ and $t_{\mathrm{po}}$ is the time required for the pull-off to take place. Such results suggest that the nature of the dissipative processes involved in the pull-off of the adhesive contacts is almost scale independent of the millimeter size down to a few tens of micrometers. In other words, the assumption that viscoelastic losses are localized near the contact line, in a small region with respect to the contact size, remains valid at the micro-scale. Moving from this consideration, a simple theoretical procedure can be derived to evaluate the evolution of the contact radius $a$ of micro-asperities contacts in the unloading phase.

\section{Declaration of competing interest}

The authors declare that they have no known competing financial interests or personal relationships that could have appeared to influence the work reported in this paper.

\section{Acknowledgments}

L.A. and G.V. acknowledge support from the Italian Ministry of Education, University and Research (MIUR) under the program "Departments of Excellence" (L.232/2016).

\section{Funding}

L.A. would like to thank for support by the project "FASTire (Foam Airless Spoked Tire): Smart Airless Tyres for Extremely-Low Rolling Resistance and Superior Passengers Comfort" funded by the Italian MIUR Progetti di Ricerca di Rilevante Interesse Nazionale (PRIN) call 2017-grant n. 2017948FEN. 

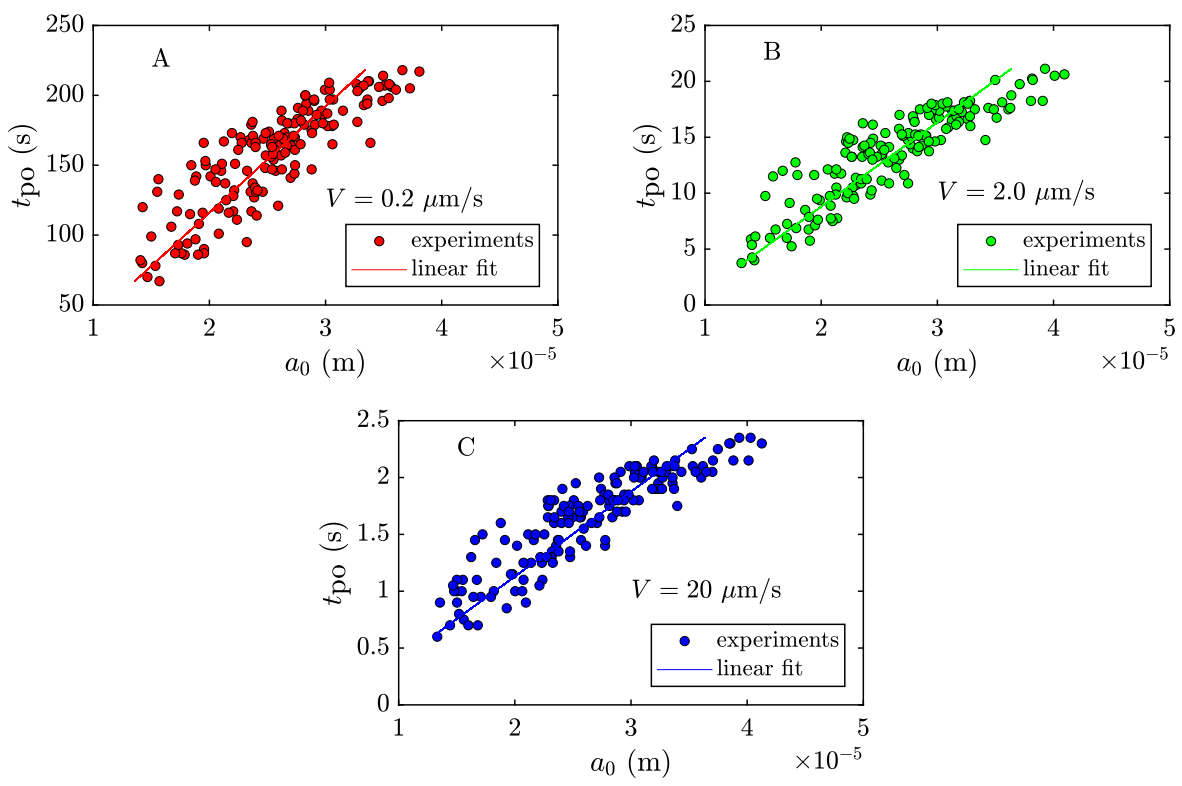

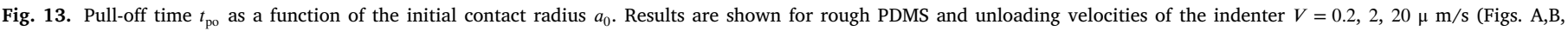
$\mathrm{C}$ respectively). Markers denote experimental data, while colored lines the corresponding linear fit; the $R^{2}$ values are $0.73,0.77$ and 0.78 (Figs. A,B, and $\mathrm{C}$ respectively).

\section{References}

Acito, V., Ciavarella, M., Prevost, A.M., Chateauminois, A., 2019. Adhesive contact of model randomly rough rubber surfaces. Tribol. Lett. 67 (2), 54. http://dx.doi.org/ 10.1007/s11249-019-1164-9.

Afferrante, L., Bottiglione, F., Putignano, C., Persson, B.N.J., Carbone, G., 2018. Elastic contact mechanics of randomly rough surfaces: An assessment of advanced asperity models and persson's theory. Tribol. Lett. 66, 75. http://dx.doi.org/10. 1007/s11249-018-1026-x.

Andrews, E.H., Kinloch, A.J., 1973. Mechanics of adhesive failure, II. Proc. R. Soc. Lond. A. Math. Phys. Sci. 332 (1590), 401-414. http://dx.doi.org/10.1098/rspa. 1973.0033.

Baek, D., Hemthavy, P., Saito, S., Takahashi, K., 2017. Evaluation of energy dissipation involving adhesion hysteresis in spherical contact between a glass lens and a PDMS block. Appl. Adhes. Sci. 5, 4. http://dx.doi.org/10.1186/s40563-017-0082-z.

Barquins, M., 1983. Influence of the stiffness of testing machines on the adherence of elastomers. J. Appl. Polym. Sci. 28 (8), 2647-2657. http://dx.doi.org/10.1002/ app.1983.070280816.

Barthel, E., Frétigny, C., 2009. Adhesive contact of elastomers: Effective adhesion energy and creep function. J. Phys. D. Appl. Phys. 42 (19), 195302. http://dx. doi.org/10.1088/0022-3727/42/19/195302.

Barthel, E., Haiat, G., 2002. Approximate model for the adhesive contact of viscoelastic spheres. Langmuir 18 (24), 9362-9370. http://dx.doi.org/10.1021/la025959+.

Charmet, J.C., Vallet, D., Barquins, M., 1998. Surface and bulk properties in adherence of elastic-viscoelastic solids. ACS Symp. Ser. http://dx.doi.org/10.1021/bk-20000741.ch003.

Deng, W., Kesari, H., 2019. Effect of machine stiffness on interpreting contact forceindentation depth curves in adhesive elastic contact experiments. J. Mech. Phys. Solids 131, 404-423. http://dx.doi.org/10.1016/j.jmps.2019.07.009.

Deplace, F., Carelli, C., Mariot, S., Retsos, H., Chateauminois, A., Ouzineb, K., Creton, C., 2009. Fine tuning the adhesive properties of a soft nanostructured adhesive with rheological measurements. J. Adhes. 85 (1), 18-54. http://dx.doi. org/10.1080/00218460902727381.

Deruelle, M., Hervet, H., Jandeau, G., Léger, L., 1998. Some remarks on JKR experiments. J. Adhes. Sci. Technol. 12 (2), 225-247. http://dx.doi.org/10.1163/ $156856198 X 00074$.

Gebeshuber, I.C., 2007. BiotRibology inspires new technologies. Nano Today 2 (5), 30-37. http://dx.doi.org/10.1016/S1748-0132(07)70141-X.

Gent, A.N., Petrich, R.P., 1969. Adhesion of viscoelastic materials to rigid substrates. Proc. R. Soc. Lond. A Math. Phys. Sci. 310 (1502), 433-448.

Gent, A.N., Schultz, J., 1972. Effect of wetting liquids on the strength of adhesion of viscoelastic materials. J. Adhes. 3 (4), 281-294. http://dx.doi.org/10.1080/ 00218467208072199.

Greenwood, J.A., Johnson, K.L., 1981. The mechanics of adhesion of viscoelastic solids. Philos. Mag. A Phys. Condens. Matter, Struct. Defects Mech. Prop. 43 (3), 697-711. http://dx.doi.org/10.1080/01418618108240402.

Haiat, G., Phan Huy, M.C., Barthel, E., 2003. The adhesive contact of viscoelastic spheres. J. Mech. Phys. Solids 51 (1), 69-99. http://dx.doi.org/10.1016/S00225096(02)00059-5.
Johnson, K.L., Kendall, K., Roberts, A.D., 1971. Surface energy and the contact of elastic solids. Proc. R. Soc. Lond. A Math. Phys. Sci. 324 (1558), 301-313. http: //dx.doi.org/10.1098/rspa.1971.0141.

Kendall, K., 1973. Shrinkage and peel strength of adhesive joints. J. Phys. D. Appl. Phys. 6 (15), 1782. http://dx.doi.org/10.1088/0022-3727/6/15/304.

Kendall, K., 2007. Molecular Adhesion and its Applications: The Sticky Universe. Springer Science \& Business Media.

Lorenz, B., Krick, B.A., Mulakaluri, N., Smolyakova, M., Dieluweit, S., Sawyer, W.G., Persson, B.N.J., 2013. Adhesion: Role of bulk viscoelasticity and surface roughness. J. Phys. Condens. Matter 25 (22), 225004. http://dx.doi.org/10.1088/0953-8984/ 25/22/225004.

Majidi, C., 2014. Soft robotics: A perspective - current trends and prospects for the future. Soft Robot. 1 (1), 5-11. http://dx.doi.org/10.1089/soro.2013.0001.

Maugis, D., Barquins, M., 1978. Fracture mechanics and the adherence of viscoelastic bodies. J. Phys. D. Appl. Phys. 11, 1989-2023. http://dx.doi.org/10.1088/00223727/11/14/011.

Muller, V.M., 1999. On the theory of pull-off of a viscoelastic sphere from a flat surface. J. Adhes. Sci. Technol. 13 (9), 999-1016.

Palchesko, R.N., Zhang, L., Sun, Y., Feinberg, A.W., 2012. Development of polydimethylsiloxane substrates with tunable elastic modulus to study cell mechanobiology in muscle and nerve. PLoS One 7 (12), http://dx.doi.org/10.1371/journal.pone. 0051499.

Ramond, G., Pastor, M., Maugis, D., Barquins, M., 1985. Mesure du module complexe par poinçonnement. Cahiers Groupe Franç. Rhéol. 6 (67), 3.

Robbe-Valloire, F., Barquins, M., 1998. Adhesive contact and kinetics of adherence between a rigid cylinder and an elastomeric solid. Int. J. Adhes. Adhes. 18 (1), 29-34. http://dx.doi.org/10.1016/S0143-7496(97)00064-X.

Roberts, A.D., 1979. Looking at rubber adhesion. Rubber Chem. Technol. 52 (1), 23-42. http://dx.doi.org/10.5254/1.3535206.

Tiwari, A., Dorogin, L., Bennett, A.I., Schulze, K.D., Sawyer, W.G., Tahir, M., Heinrich, G., Persson, B.N.J., 2017. The effect of surface roughness and viscoelasticity on rubber adhesion. Soft Matter 13 (19), 3602-3621. http://dx.doi.org/10.1039/ c7sm00177k.

Violano, G., Afferrante, L., 2019a. Adhesion of compliant spheres: An experimental investigation. In: Procedia Structural Integrity, Vol. 24. pp. 251-258. http://dx. doi.org/10.1016/j.prostr.2020.02.022.

Violano, G., Afferrante, L., 2019b. On DMT methods to calculate adhesion in rough contacts. Tribol. Int. 130, 36-42. http://dx.doi.org/10.1016/j.triboint.2018.09.004.

Violano, G., Afferrante, L., 2019c. Modeling the adhesive contact of rough soft media with an advanced asperity model. Tribol. Lett. 67 (4), 119. http://dx.doi.org/10. 1007/s11249-019-1232-1.

Williams, M.L., Landel, R.F., Ferry, J.D., 1955. The temperature dependence of relaxation mechanisms in amorphous polymers and other glass-forming liquids. $\mathrm{J}$. Am. Chem. Soc. (14), 3701-3707. http://dx.doi.org/10.1021/ja01619a008.

Yashima, S., Romero, V., Wandersman, E., Frétigny, C., Chaudhury, M.K., Chateauminois, A., Prevost, A.M., 2015. Normal contact and friction of rubber with model randomly rough surfaces. Soft Matter 11 (5), 871-881. http://dx.doi.org/10.1039/ c4sm02346c. 